\title{
Herausforderungen bei der Charakterisierung von Karstgrundwasserleitern
}

\author{
Tobias Geyer • Nico Goldscheider
}

Online veröffentlicht: 23.01.2014

(C) Springer-Verlag Berlin Heidelberg 2013

Die nachhaltige Bewirtschaftung von Karstwasserressourcen erfordert systembeschreibende Charakterisierungsansätze und Vorhersagewerkzeuge. Traditionelle hydrogeologische Geländeuntersuchungen und Modelltechniken sind häufig auf poröse Medien fokussiert und vernachlässigen stark anisotrope Strukturelemente, die neben nichtlinearen Strömungsregimes auch über große Skalenbereiche zu komplexen hydraulischen Parameterfeldern führen.

Die Parametrisierung numerischer Modelle in der Karsthydrogeologie erfordert deshalb eine differenzierte Herangehensweise. Während die hydraulischen Kennwerte der geklüfteten Matrix auf der lokalen Skala relativ einfach durch Bohrlochversuche bestimmt werden können, ist es fast unmöglich, repräsentative hydraulische Kennwerte durch kontrollierte Experimente auf der Einzugsgebietsskala zu ermitteln. Dies liegt darin begründet, dass Karströhren eine entscheidende Funktion für die einzugsgebietsweite Entwässerung des Untergrundes besitzen, aufgrund ihres geringen Volumenanteils am Gesamtsystem jedoch nur selten durch Bohrungen erfasst werden. Markierungsversuche sind bewährte Werkzeuge für die Charakterisierung des hochdurchlässigen Röhrensystems. Untersuchungen von Quellschüttung und von anderen Quellparametern erlauben zusätzlich die integrale einzugsgebietsweite Untersuchung der Grundwasserdynamik und können deshalb wichtige Informationen für die Kalibrie-

\footnotetext{
T. Geyer $(\bowtie)$

Landesamt für Geologie, Rohstoffe und Bergbau

im Regierungspräsidium Freiburg i. Br.,

Albertstr. 5, 79104 Freiburg, Deutschland

E-Mail: tobias.geyer@rpf.bwl.de

N. Goldscheider

Institut für Angewandte Geowissenschaften,

KIT - Karlsruher Institut für Technologie,

Kaiserstr. 12, 76131 Karlsruhe, Deutschland

E-Mail: nico.goldscheider@kit.edu
}

rung numerischer Modelle liefern. In den aktuellen Themenheften sind dazu verschiedene Beispiele dokumentiert.

Zudem können neue Methoden im Bereich der Messtechnik und Analytik einen wichtigen Beitrag zur Charakterisierung der Systeme liefern. Die Anwendung von Datenloggern erlaubt z. B. die zeitlich hochaufgelöste Erfassung der Dynamik von Karstquellen als Resultat schneller Strömungs- und Transportprozesse. Für die Untersuchung der langsamen mobilen Fließkomponente wurde in der Vergangenheit häufig Tritium verwendet, dessen heutige Anwendung jedoch stark erschwert ist. Die Anwendung und Vergleichbarkeit alternativer Umwelttracer (z. B. Gastracer) in der Karsthydrogeologie ist deshalb zu prüfen. Verschiedene aktuelle Studien zeigen zudem, dass andere Indikatoren im Quellwasser, wie z. B. organische Spurenstoffe und Partikel, einen wichtigen Beitrag für die Bestimmung der Herkunft und Verweilzeit des Wassers leisten können. Die Verweilzeitenverteilung im Fließsystem wird neben der Interaktion von schnellen und langsamen Fließkomponenten auch von der zeitlichen und räumlichen Verteilung der Grundwasserneubildung gesteuert. Während die Neubildungsmenge relativ genau und flächendeckend ermittelt werden kann, z. B. mit Bodenwasserhaushaltsmodellen, fehlen immer noch prozesstreue Ansätze zur Simulation der Wasserperkolation durch die ungesättigte Festgesteinszone.

Die Charakterisierung und Modellierung von Karstgrundwasserleitern ist somit aufgrund ihrer komplexen Struktur- und Prozessvielfalt immer noch eine große Herausforderung und wird auch in Zukunft ein spannendes Feld der Hydrogeologie bleiben.

Das vorliegende und das vorherige Themenheft präsentieren Beiträge zur Karsthydrogeologie aus dem deutschsprachigen Raum (D-A-CH). Es sind aber auch internationale Autoren und Studien vertreten. Die Themenhefte sind daher auch ein Beitrag zu den Aktivitäten der IAH Karstkommission (www.iah.org/karst). 